\title{
An Unusual Case of Ingested Foreign Body with Disastrous Outcome: A Case Report
}

\author{
Moumita Acharya $^{1 *}$, Sibnath Mandal ${ }^{2}$, Kankana Rana $^{2}$
}

${ }^{1}$ SR, Calcutta National Medical College \& Hospital, 24 Gorachand Road, Beniapukur, Kolkata, West Bengal India

${ }^{2}$ Consultant, Peerless Hospital, 360 Panchasayar, Kolkata 700094, West Bengal, India

DOI: $\underline{10.36347 / \text { sasjs.2020.v06i10.001 }}$

| Received: 09.09.2020 | Accepted: 12.10.2020 | Published: 17.10.2020

*Corresponding author: Dr. Moumita Acharya

Foreign bodies (FB) in digestive tracts are not unusual, usually children present with accidental ingestion. We here describe a case of 42 years old woman presenting with accidental ingestion of sharp-edged FB, which had to be removed by anterior resection of colon. Ingested sharp FBs pose risks of perforation and bowel obstruction. Conservative approach by tracking FB migration on serial imaging can be depended on provided there are no features of perforation/obstruction. Minimally invasive techniques like gastroscopy, colonoscopy is a safe, efficient means of removal but conventional surgical methods (colotomy, segmental resections etc) may also be required if minimally invasive methods fail. Appropriate technique will depend on characteristics of FB and patients presenting features.

Keywords: Accidental, foreign body, sigmoid, anterior resection.

Copyright $(\mathcal{C} 2020$ The Author(s): This is an open-access article distributed under the terms of the Creative Commons Attribution 4.0 International License (CC BY-NC 4.0) which permits unrestricted use, distribution, and reproduction in any medium for non-commercial use provided the original author and source are credited.

\section{INTRODUCTION}

Foreign bodies in the digestive tract are unusual, being either swallowed accidentally in children or sometimes inserted from the anal end for selfgratification. Swallowed objects generally get stuck in the cricopharynx, being the narrowest part of the upper digestive tract. Further down, they may get stuck at the lower oesophageal sphincter or lower down proximal to the ileocecal junction.

Here we present an unusual case of an accidentally ingested sharp foreign body (tablet in-foil) causing no features of upper GI obstruction, perforation and finally lodging in the rectosigmoid junction with localised perforation. It was retrieved 10 days later in intact form by anterior resection.

\section{CASE REPORT}

A 42 year old lady, hypertensive, presented to the ER with 7days history of accidental ingestion of calcium tablets in-foil wrapper. On non passage of the foil with stool, over the next few days, the patient sought medical help. She was otherwise symptom free with no abdominal pain or distension, no vomiting, no history of bleeding per rectum or melena. Tolerating diet and passing stool and flatus normally. On admission she was afebrile, hemodynamically stable. Routine blood tests within normal levels. Serial imaging revealed the radio opaque foreign body descending but not beyond a point in the pelvis.

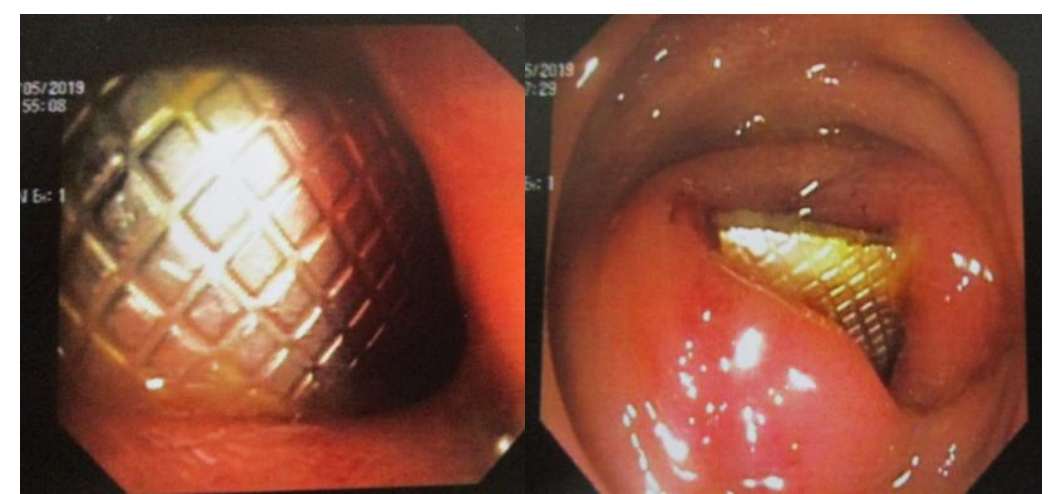

Flexible sigmoidoscopy revealed rectum is normal. Foreign body (tablet foil) seen at distal sigmoid colon. Sharp edged. Failed attempts at retrieval. 


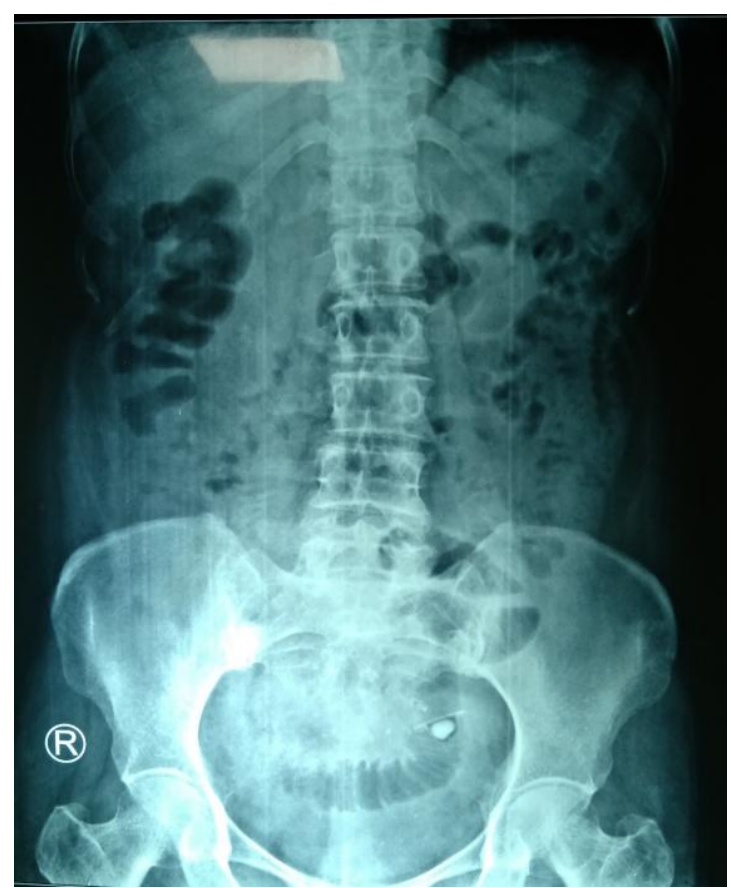

Straight Xray abdomen showed radio opaque shadow in left hemipelvis near the Left Sacroiliac joint

With failure of conservative measures, the patient was taken up for Colonoscopy \& proceed. On table colonoscopy failed to remove the impacted foreign body. Midline laparotomy done. Thick walled rectosigmoid junction with localised perforation walled off with small bowel seen. As bowel was thick walled, oedematous, closing off the perforation is not feasible.
Anterior resection done. Diseased bowel resected using TA 45. Attempted anastomosis using $29 \mathrm{~mm}$ circular gun, abandoned. Another TA 60 used to resect upper rectum distal to attempted anastomosis. Circular gun $32 \mathrm{~mm}$ used for anastomosis. Leak test done; no leak. Drain given. Post operative recovery was slow but uneventful.

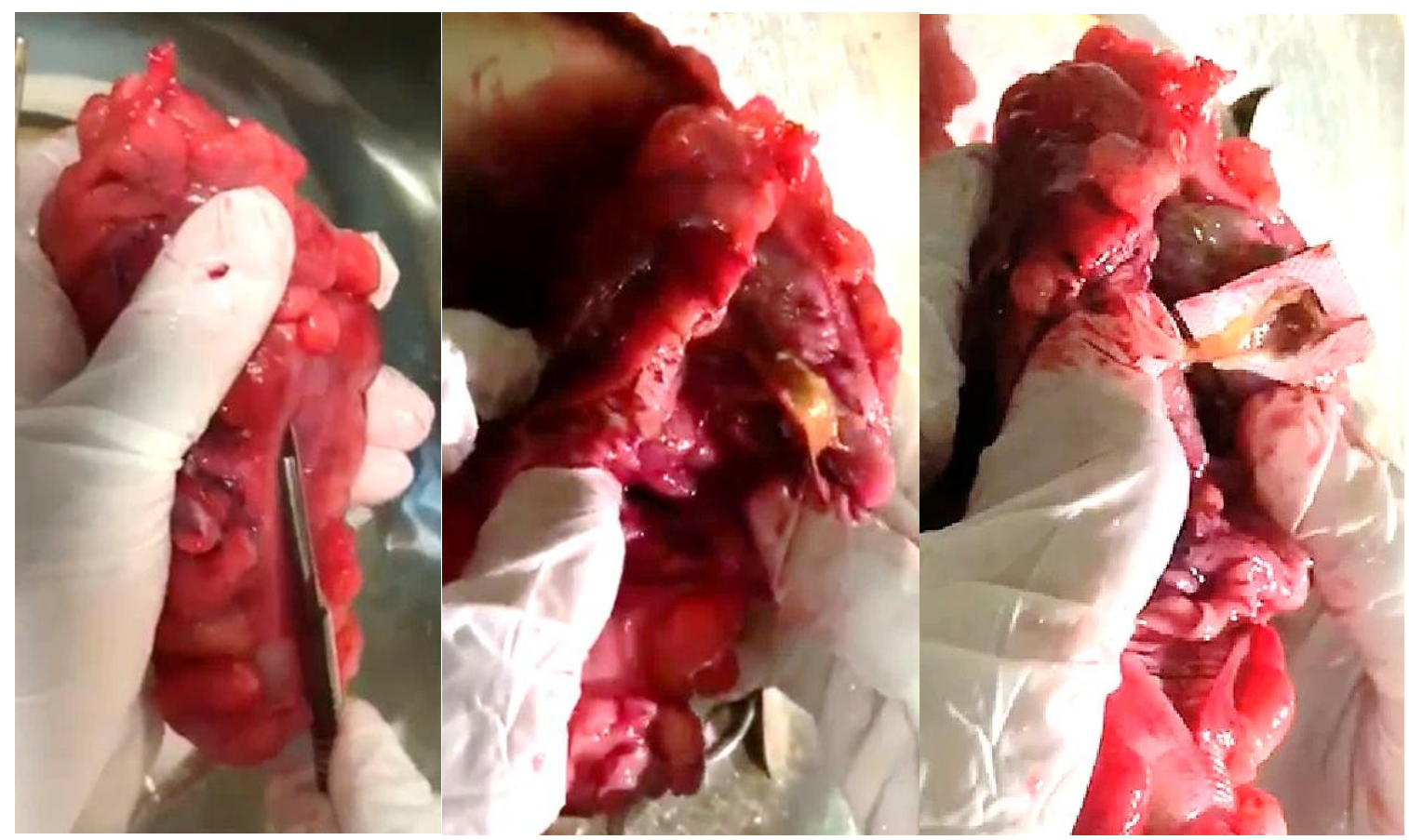

\section{DISCUSSION}

It is strange how a sharp foreign body caused no injuries else where and got impacted with localised perforation in the sigmoid colon. Some reported cases of foreign bodies in colon with no features of perforation are:

a) Embedded dentures in caecum in 84year old gentleman [1] 
b) Self expulsion of Gauze left behind from prior cholecystectomy, in 40 year old lady [2]

c) Impacted fishbone at rectosigmoid junction in 32year old gentleman [3]

Three management modalities have been described for FB ingestion and impaction: Observation, Endoscopy and Surgery [4]. Observation is possible when objects are small $(<4 \mathrm{~cm})$, provide no trauma to GI tract, and the location is distal to ligament of Treitz (uncomplicated lower GI foreign bodies) [4]. Endoscopy is preferred modality for uncomplicated impaction especially upper GI tract [5]. Surgery is warranted in complicated cases $(<1 \%)[4,6]$.

\section{CONCLUSION}

This is a unique case report of an accidentally ingested sharp foreign body (with 3 sharp edges and 2 sharp sides) causing no features of intestinal obstruction, perforation and finally lodging in the rectosigmoid junction causing localised perforation. It was eventually retrieved in intact form 10days later by anterior resection.

\section{Declaration of Patient Consent}

The authors certify that they have obtained all appropriate patient consent forms. In the form the patient has given her consent for her images and other clinical information to be reported in the journal. The patient understand that her name and initials will not be published and due efforts will be made to conceal her identity, but anonymity cannot be guaranteed.

\section{Financial support and sponsorship: Nil.}

Conflicts of interest: There are no conflicts of interest

\section{REFERENCES}

1. Ghanimeh MA, Abughanimeh $\mathrm{O}$, Albadarin S, Kaddourah O, Helzberg JH. Case reports in gastrointestinal medicine. 2018. article ID 2154879

2. Brown S. Foreign body in the colon: case report. Radiology. 1927; 9:2, 164-166

3. Peixoto A, Silva M, Órfão B, Macedo G. Fishbone impaction in the colon. Revista Española de Enfermedades Digestivas. 2017 Jun;109(6):460-1.

4. Gachabayov M, Isaev M, Orujova L, Isaev E, Yaskin E, Neronov D. Swallowed dentures: two cases and a review. Annals of medicine and surgery. 2015; 4:407-413

5. Bandyapadhyay SN, Das S, Das SK, Mandal A. Impacted dentures in in the esophagus. The journal of laryngology \& otology. 2014; 128(41):468-474

6. Ikenberry SO, Jue TL, Anderson MA. Management of ingested foreign bodies and food impactions. Gastrointestinal endoscopy. 2011; 73(6):1085-1091 